\title{
Canine Scent Detection of Lung Cancer: Preliminary Results
}

\author{
Albertini $\mathbf{M}^{1 *}$, Mazzola $\mathrm{S}^{1}$, Sincovich $\mathbf{M}^{2}$ and Pirrone $\mathrm{F}^{2}$ \\ ${ }^{1}$ Department of Veterinary Medicine, University of Milan, Italy \\ ${ }^{2}$ Freelance Dog Trainer
}

Research Article

Volume 1 Issue 4

Received Date: September 21, 2016

Published Date: October 21, 2016

*Corresponding author: Albertini M, Department of Veterinary Medicine, University of Milan, Italy, Tel: 00390250318128; Email: mariangela.albertini@unimi.it

\section{Abstract}

Several researches have evidenced that cancer cells can produce volatile organic compounds (VOCs) which are released not only in breath but also in other organic fluids, such as blood and urine. This study has evaluated the olfactory capability of trained dogs to detect human lung cancer VOCs in urine. We recruited 150 subjects from European Institute of Oncology (IEO) divided into three groups: 57 patients with lung cancer (group 1); 38 patients with lung disease, other than cancer (group 2); 55 healthy control subjects (group 3).The results are referred to the last 45 days of training, and evidenced that dogs reached a mean success rate that exceeded $80 \%$, with a sensitivity of 0,72 and a specificity of 0,94 for two out of three dogs enrolled. The important novelty is that dogs can discriminate lung cancer not only from healthy subjects, but also from patients with other lung diseases. The results obtained so far are encouraging and lead us to persevere with the training session in order to improve the success rate, reaching values as close as possible to $100 \%$. If so, we believe that, in the future, dogs may be used to perform early diagnostic tests, useful in improving the chances of survival in cases of human lung cancer.

\section{Introduction}

Lung cancer (Lc) in humans is the leading cause of cancer-related deaths in the world. The 5 years survival rate is $54 \%$ for cases diagnosed when the disease is still localized, but only $15 \%$ of lung cancers are identified at this early stage [1], due to the limited capabilities of existing diagnostic methods. Therefore, early detection of lung cancer is a desirable goal, because it often permits an improvement of the prognosis and a longer survival. In 1985, Gordon SM et al. have demonstrated that lung cancer cell lines can produce specific volatile organic compounds (VOCs) which are released into breath [2]. Some of these VOCs are likely to have distinctive odors which can provide information for diagnostic purposes [2]. Actually, the domestic dog is widely considered as a fast, mobile and relatively accurate detection system available for locating odorous substances. This suggests that dogs can provide a contribution also in the field of oncology. Even when present in minute quantities, VOCs are believed to be detectable by dogs, with their exceptional olfactory acuity [3,4]. In 2004, Willis CM et al. [5] published the first paper showing that dogs can detect bladder cancer by olfactory means, and afterwards, a number of studies examining the utility of analyzing VOCs in exhaled breath using dogs have been carried out and have shown promising - but still not definite - results $[6,7]$. Unfortunately, collecting, handling, storing, concentrating and analyzing breath samples are troublesome and technically challenging. A partial solution to these problems would be the use of a much more convenient source of volatiles, such as urine samples. Based on this consideration, Matsumura et al. [8] were able to identify volatile chemical signatures in urine of mouse models of lung cancer using rigorous experimental behavioral and analytic techniques, establishing the feasibility of using urinary volatiles to detect lung cancer. 
Our study aimed to train dogs for human lung cancer related VOC detection in urine, in order to make an early diagnosis and to improve the prognosis, contributing to reduce cancer mortality.

\section{Materials and Methods}

\section{Subjects}

Human volunteers were recruited by European Institute of Oncology (IEO). Eligible patients were men and women aged between 50 and 80 years old, healthy or with a recent conventional diagnosis of lung cancer or other types of pulmonary disease. In case of lung cancer, patients were referred immediately after definitive diagnosis, to avoid potential chemotherapy-induced alteration of the profile of urinary VOCs. None of the patients had a previous historyof cancer within five years.

Healthy subjects, with the same age range, were selected as the control group. None of them had a referred history of clinically significant pulmonary disease. In order to confirm the absence of oncological findings, they were required to have had a recent (within the previous 6 months) negative chest x-ray or CT scan.

Drugs, menstrual cycle, ethnicity, diet, alcohol consumption, smoking habits and exposure to chemicals were not considered exclusion criteria. However, these factors were recorded in order to analyze their possible influence on dog's signaling. All volunteers were provided with appropriate information concerning the study and a written informed consent were obtained from them all.

Subjects were divided into three groups:

Group 1) patients with lung cancer (n. 57)

Group 2) patients with lung disease, other than cancer (n. 38)

Group 3) healthy control subjects (n. 55).

\section{Sampling of urine}

Each volunteer donated at least $20 \mathrm{ml}$ of urine, which was placed in a sterile container. Urine samples were refrigerated within $45 \mathrm{~min}$ and frozen 2-32 hours later, as $1 \mathrm{ml}$ aliquots in hermetically sealed tubes. As reported in literature [6], these samples can be stored at $-40^{\circ} \mathrm{C}$ for up to five months. For presentation to the dogs, samples were defrosted and immediately used, in a wet state.

\section{Dog training}

We employed three family dogs: two female Belgian Malino is shepherd dogs, Bloom and Dixie, 3 and 5 years old respectively, and a 3-year-old female mixed breed dog, Helix. They were trained by clicker training method (operant conditioning) with positive reinforcement (food).

During training, animals worked with samples taken from patients of all three groups. Training sessions were held twice a week, in a dedicated facility of the Veterinary Department of the University of Milan, where no other animal had access, in order to avoid odor bias. The room was well aerated and conditioned to maintain optimal temperature and humidity conditions, therefore avoiding climate interference.

The six sample stations, designed and constructed of stainless steel, were positioned in a single straight line as in Figure 1. The training consisted of two sessions of six runs. In each run, samples tubes were selected to compose six-slot panels so that 1 sample of Group 1 and at least 1 sample of Group 2 were compared with samples of healthy subjects, possibly varying the identity of the donors. The positions of the samples were randomly changed throughout the successive trials; thus, the dog's choices could not be driven by any memory interference. Moreover, since each dog carried out two sessions per day, at the end of each session the six-slot panels were changed and the sample station perfectly cleaned with a vapor machine. Dogs were asked to indicate the correct responses by sitting directly in front of the station containing the cancer sample.

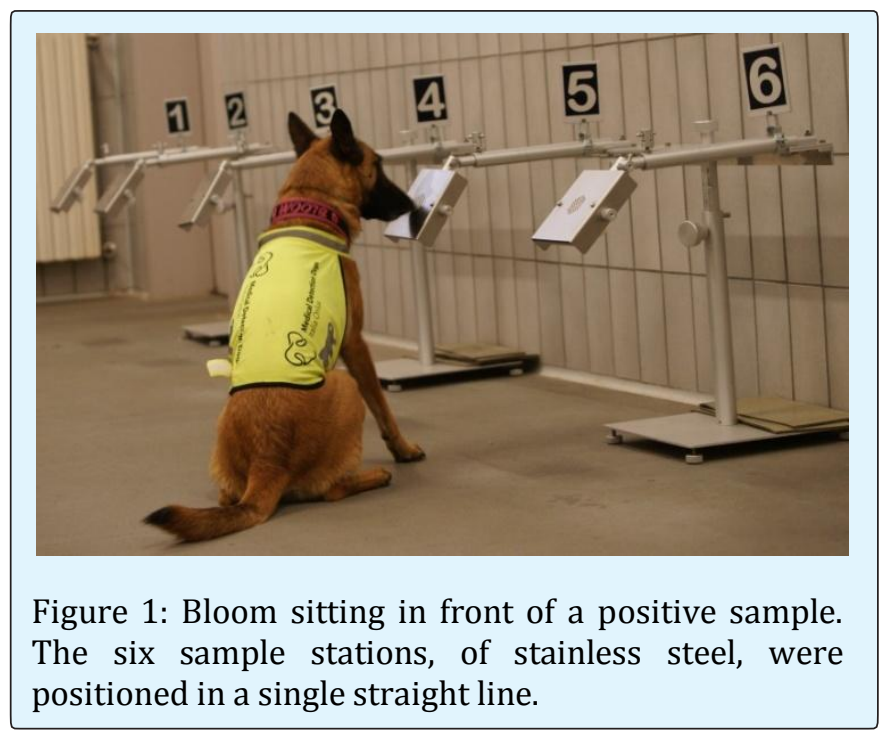

All the sessions were videotaped, for accurate analysis of canine responses and behavior. High standards of ethics were respected in study design and conduction, to guarantee dog's psychophysical wellbeing. 


\section{Statistical Analysis and Results}

We decided to analyze the last 45 days of training, i.e. the last six months, during which the dog handler was blinded to knowledge of the target sample. Statistical analysis was performed using IBM SPSS Statistics for Windows, version 22.0 (Armonk, NY: IBM Corp). The primary outcome measure was the mean \pm S.E proportion of successes for each dog. The Kruskal-Wallis test was used to detect statistically significant differences in the success rates of the three dogs throughout the entire training cycle.

Diagnostic accuracy was calculated as sensitivity and specificity of the dog's indication of samples compared with the true diagnosis confirmed by TAC. Thus, the sensitivity refers to the conditional probability of the dog indicating cancer when the condition was present and specificity refers to the conditional probability of the dog ignoring a sample from a healthy donor. Both sensitivity and specificity were expressed as proportions. Point estimates were calculated with $95 \%$ confidence intervals. The probability of a perfect test run (finding the right sample and ignoring the controls) by chance was $1 / 6$ $(17 \%)$. All statistical tests were 2 -sided with exact $\mathrm{p}<0.05$ considered statistically significant. In Figure 2 is reported all dog's mean success rate, during the last 45 days of training, which shows an improvement trend that exceeds $80 \%$ at the end of the period considered. Dogs' sensitivity and specificity are reported in Table 1. "DOG ID" was a significant positive predictor for successful outcomes (Table 2). As shown by the Exp (B) coefficient in the logistic regression, both Bloom and Helix were about 2.5 times more likely to correctly locate the cancer sample and ignoring all 5 control samples.

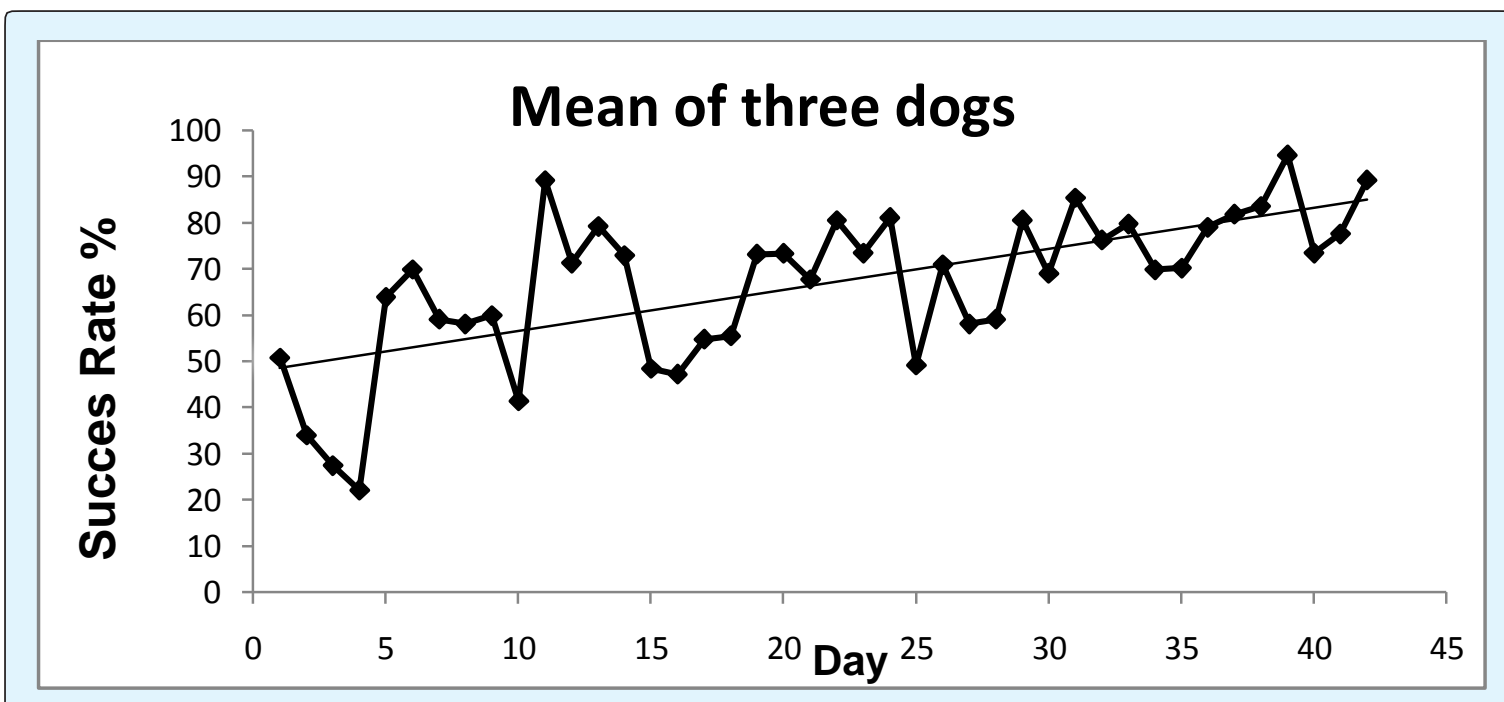

Figure 2: Dog's mean success rate trend during training.

\begin{tabular}{|c|c|c|c|c|}
\hline \multicolumn{2}{|c|}{ Dog ID } & \multirow{2}{*}{ Estimate } & \multicolumn{2}{c|}{ 95\% } \\
\cline { 3 - 5 } Bloom & Sensitivity & 0,782 & 0,670 & Upper \\
\cline { 2 - 5 } & Specificity & 0,956 & 0,908 & 0,899 \\
\hline \multirow{3}{*}{ Helix } & Sensitivity & 0,650 & 0,515 & 1,011 \\
\cline { 2 - 5 } & Specificity & 0,930 & 0,857 & 0,785 \\
\hline \multirow{3}{*}{ Dixie } & Sensitivity & 0,595 & 0,452 & 1,002 \\
\cline { 2 - 5 } & Specificity & 0,362 & 0,838 & 1,002 \\
\hline
\end{tabular}

Table 1: Diagnostic test accuracy. 


\section{Open Access Journal of Veterinary Science \& Research}

\begin{tabular}{|c|c|c|c|c|c|}
\hline Factors & $\mathbf{P}$ & $\mathbf{E x p ( B )}$ & \multicolumn{2}{|c|}{$\mathbf{9 5 \%}$ CI for Exp(B) } & $\begin{array}{c}\text { Hosmer-Lemeshow } \\
\text { significance test }\end{array}$ \\
\hline Dog (Bloom) & 0,001 & 2,598 & Lower & Upper & 0,240 \\
\cline { 4 - 5 } & & 1,887 & 3,876 & \\
\hline Dog (Helix) & 0,001 & 2,395 & 1,808 & 3,172 & \\
\hline
\end{tabular}

Table 2: Logistic regression models predicting correct discrimination.

Only factors for which a statistically significant difference emerged are reported. Significance, $\mathrm{P}<0.05$. Exp(B), Exponentiation of the B coefficient (odds ratio). $\mathrm{CI}$, confidence interval.

\section{Discussion}

Lung cancer continues to represent a heavy burden for health care systems worldwide. This study evidenced that Lc gives a VOC-related odor signature to urine, which can be detected by dogs. This preliminary result shows that canine olfactory capability may be considered a valid diagnostic method for early detection of Lc in humans, with the advantage of being a fast, non-invasive, painless and inexpensive method. Moreover, to the best of our knowledge, this is the first study evidencing that dogs can discriminate urine of patients with lung cancer not only from healthy subjects, but also from patients with other types of pulmonary disease.

The hypothesis that dogs may be able to detect cancer in humans on the basis of specific odor find its roots in 1989, when Williams and Pembroke described, for the first time, the case of a 44-year-old woman who became aware of a melanoma on her left thigh after her untrained female dog began sniffing intensely and repeatedly at the lesion through her clothing [9]. Since then, similar anecdotal events have been reported. Although unsupported by experimental evidence, they had the merit of drawing medical community's attention to the concept that dogs can "smell" cancer in patients. As time goes by, intriguing evidence emerged that melanoma and bladder cancers could be detected by dogs through their scenting abilities [10]. In 2003, John Church brought together a team of doctors, dog trainers and scientists and in 2004 a preliminary proof of principle study was completed [5]. This study provided the first piece of experimental evidence to show that dogs can detect cancer by olfactory means and was the first clinically robust trial to be completed and published in the world.

Since 1982, researches were also conducted to develop sensor arrays, commonly referred to as "electronic noses", which could identify VOCs linked to cancer since early stages using breath samples [11-13]. However their applicability is limited because an optimized sample collection is necessary, the instruments are very sensitive and no lung cancer-specific VOCs have been identified to date [14]. Moreover, patients are required not to smoke and fast before breath samples are taken. Finally, it has been shown that measuring VOCs with electronic nose has not yet been standardized, and the set-up significantly affects the results [15]. Canine scent detection may help overcome some of these drawbacks, due to the extraordinary dog's ability to detect and discriminate a specific odor among a myriad of odorant components.

In our study, the dog's mean success rate has improved during the training period, reaching a value that exceeded $80 \%$. We are confident that success rate can be further improved after an adequate ongoing period of training. If the next phases of this study will confirm these results, the integration of sniffer dogs into research and prevention strategies will turn out to be a useful tool for early diagnosis of lung cancer, giving the possibility to improve patient survival. Moreover, the ability of dogs to detect cancer can be usedas a friendly message to the public, encouraging people to undergo medical examination even in the absence of any signs or symptoms.

\section{Acknowledgments}

This work was supported by Fondazione Cariplo, Grant No. 2014-0105. We want to thank the Division of Thoracic Surgery of the European Institute of Oncology, Milan, Italy, who enrolled the patients and contributed in the sampling, collection and storing of urine samples. We are very grateful to Aldo La Spina and the charity Medical Detection Dogs Italy for their support.

\section{References}

1. Gasparri R, Santonico M, Valentini C, Sedda G, Borri A, et al. (2016) Volatile signature for the early diagnosis of lung cancer. J Breath Res 10(1): 016007. 


\section{Open Access Journal of Veterinary Science \& Research}

2. Gordon SM, Szidon JP, Krotoszynski BK, Gibbons RD, O'Neill HJ (1985) Volatile organic compounds in exhaled air from patients with lung cancer. Clin Chem 31(8): 1278-1282.

3. Schoon GAA, De Bruin JC (1994) The ability of dogs to recognise and cross-match human odours. Forensic Sci Int 69(2): 111-118.

4. Lorenzo N, Wan TL, Harper RJ, Hsu YL, Chow M, et al. (2003) Laboratory and field experiments used to identify Canis lupus var. familiaris active odor signature chemicals from drugs, explosives, and humans. Anal Bioanal Chem 376(8): 1212-1224.

5. Willis CM, Church SM, Guest CM, Cook WA, McCarthy $\mathrm{N}$, et al. (2004) Olfactory detection of human bladder cancer by dogs: proof of principle study. BMJ 329(7468): 712.

6. McCulloch M, Jezierski T, Broffman M, Hubbard A, Turner K, et al. (2006) Diagnostic accuracy of canine scent detection in early- and late-stage lung and breast cancers. Integr Cancer Ther 5(1): 30-39.

7. Ehmann R, Boedeker F, Friedrich U, Sagert J, Dippon J, et al. (2012) Canine scent detection in the diagnosis of lung cancer: revisiting a puzzling phenomenon. Eur Respir J 39(3): 669-676.

8. Matsumura K, Opiekun M, Oka H, Vachani A, Albelda SM, et al. (2010) Urinary volatile compounds as biomarkers for lung cancer: a proof of principle study using odor signatures in mouse models of lung cancer. PLoS ONE 5(1): e8819.

9. Williams H, Pembroke A (1989) Sniffer dogs in the melanoma clinic? Lancet 1(8640): 734.

10. Church J, Williams H (2001) Another sniffer dog for the clinic? Lancet 358(9285): 930.

11. Persaud K, Dodd G (1982) Analysis of discrimination mechanisms in the mammalian olfactory system using a model nose. Nature 299(5881): 352-355.

12. Horvàth I, Làzàr Z, Gyulai N, Kollai M, Losonczy G (2009) Exhaled biomarkers in lung cancer. Eur Respir J 34(1): 261-275.

13. Peng G, Hakim M, Broza YY, Billan S, Abdah-Bortnyak $\mathrm{R}$, et al. (2010) Detection of lung, breast, colorectal, and prostate cancer from exhaled breath using a single array of nanosensors. Br J Cancer 103(4): 542551.

14. D’Amico A, Pennazza G, Santonico M, Martinelli E, Roscioni C, et al. (2010) An investigation on electronic nose diagnosis of lung cancer. Lung Cancer 68(2): 170-176.

15. Koczulla R, Hattesohl A, Biller H, Hofbauer J, Hohlfeld J, et al. (2011) Comparison of four identical electronic noses and three measurements set-ups. Pneumologie 65(8): 465-470. 